ARTICLE

\title{
Reprogramming bacterial protein organelles as a nanoreactor for hydrogen production
}

Tianpei Li1 ${ }^{1,2,3,4}$, Qiuyao Jiang ${ }^{1}$, Jiafeng Huang ${ }^{1,5}$, Catherine M. Aitchison ${ }^{6}$, Fang Huang ${ }^{1}$, Mengru Yang ${ }^{1}$, Gregory F. Dykes (1D ${ }^{1}$, Hai-Lun He ${ }^{5}$, Qiang Wang ${ }^{4,7 凶}$, Reiner Sebastian Sprick ${ }^{6}$, Andrew I. Cooper (1) ${ }^{6} \&$ Lu-Ning Liu (iD) $1,8 \times$

Compartmentalization is a ubiquitous building principle in cells, which permits segregation of biological elements and reactions. The carboxysome is a specialized bacterial organelle that encapsulates enzymes into a virus-like protein shell and plays essential roles in photosynthetic carbon fixation. The naturally designed architecture, semi-permeability, and catalytic improvement of carboxysomes have inspired rational design and engineering of new nanomaterials to incorporate desired enzymes into the protein shell for enhanced catalytic performance. Here, we build large, intact carboxysome shells (over $90 \mathrm{~nm}$ in diameter) in the industrial microorganism Escherichia coli by expressing a set of carboxysome protein-encoding genes. We develop strategies for enzyme activation, shell self-assembly, and cargo encapsulation to construct a robust nanoreactor that incorporates catalytically active [FeFe]hydrogenases and functional partners within the empty shell for the production of hydrogen. We show that shell encapsulation and the internal microenvironment of the new catalyst facilitate hydrogen production of the encapsulated oxygen-sensitive hydrogenases. The study provides insights into the assembly and formation of carboxysomes and paves the way for engineering carboxysome shell-based nanoreactors to recruit specific enzymes for diverse catalytic reactions.

\footnotetext{
${ }^{1}$ Institute of Systems, Molecular and Integrative Biology, University of Liverpool, Liverpool L69 7ZB, UK. ${ }^{2}$ Key Laboratory of Algal Biology, Institute of Hydrobiology, Chinese Academy of Sciences, 430072 Wuhan, China. ${ }^{3}$ University of Chinese Academy of Sciences, 100049 Beijing, China. ${ }^{4}$ State Key Laboratory of Crop Stress Adaptation and Improvement, School of Life Sciences, Henan University, 475004 Kaifeng, China. ${ }^{5}$ School of Life Sciences, Central South University, Changsha, China. ${ }^{6}$ Materials Innovation Factory and Department of Chemistry, University of Liverpool, Liverpool L7 3NY, UK. ${ }^{7}$ Innovation Academy for Seed Design, Chinese Academy of Sciences, Beijing, China. ${ }^{8}$ College of Marine Life Sciences, and Frontiers Science Center for Deep Ocean

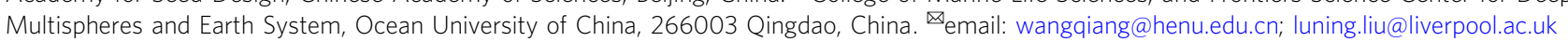




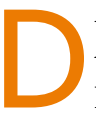
ue to climate change, there is a pressing need to reduce the emission of carbon dioxide from burning fossil fuels. Hydrogen has been identified as a potential replacement for sustainable and clean energy sources, since consuming hydrogen generates only water as the byproduct and the highenergy content of the $\mathrm{H}-\mathrm{H}$ bond making hydrogen an efficient means of storing energy ${ }^{1,2}$. However, most hydrogen is currently produced non-renewably from steam-reforming processes, which release a substantial amount of carbon dioxide ${ }^{3}$. Therefore, increasing attention has been paid to the development of novel hydrogen-forming catalysts in chemistry and biology 4

Hydrogenases are enzymes that catalyze the generation and conversion of hydrogen. The exceptional catalytic activity and efficiency of hydrogenases make them valid candidates for biological hydrogen production in synthetic engineering ${ }^{5}$. Three distinct classes of hydrogenases have been discovered: [Fe]hydrogenases, $[\mathrm{NiFe}]$-hydrogenases and $[\mathrm{FeFe}]$-hydrogenases ${ }^{6}$. Among these hydrogenases, $[\mathrm{FeFe}]$-hydrogenases are the most efficient enzymes for catalytic hydrogen turnover, and arguably the promising biocatalysts for hydrogen production. However, a major drawback of [FeFe]-hydrogenases is their extreme oxygen sensitivity and irreversible inactivation by oxygen ${ }^{7,8}$. To overcome these limitations, an innovative strategy is to incorporate hydrogenases to synthetic scaffolding platforms, such as polymersomes, liposomes and virus-like particles, ensuring the encapsulation and condensation of enzymes as well as the modulation of enzymatic activities ${ }^{9,10}$. It has been recently shown that the protein-based capsid of bacteriophage P22 could encapsulate oxygen-tolerant [NiFe]-hydrogenases and offer stability and protection to the enzymes; the elevated local concentration of hydrogenases in the nanoreactor led to improved hydrogenproducing activities ${ }^{11}$. This represents a step towards synthetically engineering large and stable nanoreactors that are capable of sequestering high copy numbers of cargo enzymes and providing selective permeability to substrates to boost enzyme activities.

In nature, many bacteria have evolved virus-like proteinaceous organelles, termed bacterial microcompartments (BMCs), to compartmentalize metabolic reactions and confines toxic/ inhibitory metabolites to mitigate the crosstalk of metabolites $^{12-14}$. BMCs encapsulate catalytic enzymes within an icosahedral shell that serves as a physical barrier to selectively mediate flux of metabolites, while condensing and protecting cargo enzymes ${ }^{15-17}$. Carboxysomes (including $\alpha$-carboxysomes and $\beta$-carboxysomes) are anabolic BMCs, functioning as the key $\mathrm{CO}_{2}$-fixing machinery in all cyanobacteria and some chemoautotrophs ${ }^{18-21}$. The primary carboxylating enzymes, ribulose-1,5-bisphosphate carboxylase oxygenase (Rubisco), and carbonic anhydrase (CA), are encased by the carboxysome shell that is constructed by diverse shell proteins through selfassembly ${ }^{21-24}$. The shell allows entry of $\mathrm{HCO}_{3}{ }^{-}$into the carboxysome interior, where CA dehydrates $\mathrm{HCO}_{3}{ }^{-}$into $\mathrm{CO}_{2}$ in the vicinity of Rubisco enzymes (Fig. 1a). The carboxysome shell is also believed to exclude passage of $\mathrm{O}_{2}$ and reduce $\mathrm{CO}_{2}$ leakage into the cytosol ${ }^{25}$, resulting in a $\mathrm{CO}_{2}$-rich microenvironment that favours the carboxylation of Rubisco, thereby enhancing carbon fixation. The intriguing features of carboxysomes-self-assembly, modularity, shell permeability, and catalytic enhancement-have made them attractive engineering objectives to supercharge cellular metabolism ${ }^{26}$. Some of these principles have been incorporated into synthetic, abiotic materials such as metal-organic frameworks to allow cooperative absorption of $\mathrm{CO}_{2}{ }^{27}$.

We reasoned that the naturally occurring microenvironment generated within the carboxysome shell might enhance the catalytic performance of [FeFe]-hydrogenases, and in general any $\mathrm{O}_{2}$-sensitive enzymes and molecules. Thus, rewiring the carboxysomal shell to encapsulate [FeFe]-hydrogenases shows promise for creating a catalyst to integrate hydrogen-producing enzymes and strengthen their oxygen tolerance for improving hydrogen production. This notion has been supported by the facts that recombinant carboxysome structures could be engineered and modulated in non-native hosts, such as Escherichia $\operatorname{coli}^{28,29}$.

Here, we generate the heterologous expression systems to produce intact, stable $\alpha$-carboxysome shells $(\sim 100 \mathrm{~nm}$ in diameter) in E. coli and then perform systematic characterizations of the assembly of the protein shells. We also define the endogenous encapsulation peptide (EP) to direct external proteins into the shell. Based on the developed expression and encapsulation systems, we recruit $[\mathrm{FeFe}]$-hydrogenases and their partners ferredoxin (Fd) from the green alga Chlamydomonas reinhardtii and ferredoxin:NADP ${ }^{+}$-oxidoreductase (FNR) from E. coli into the shell, to create a catalytically functional hydrogen-producing nanoreactor in E. coli. Compared to free hydrogenases, this hydrogenase-encapsulating catalyst possesses an improved performance for $\mathrm{H}_{2}$ production and also has superior to $\mathrm{O}_{2}$ resistance. Our study provides insight into the self-assembly and selective permeability of a-carboxysomes, which is extendable to other protein organelles. It also informs bioinspired design and engineering of metabolic nanoreactors for a diverse range of functions.

\section{Results and discussion}

Generation of an entire synthetic a-carboxysome shell. According to the types of encapsulated Rubisco, carboxysomes can be categorized into $\alpha$-carboxysomes and $\beta$-carboxysomes ${ }^{19,30}$. Distinct from the 'inside out' de novo assembly of $\beta$ carboxysomes $^{31-33}$, the assembly of $\alpha$-carboxysomes appears to start from shell formation ${ }^{34}$ or a simultaneous shell-interior assembly ${ }^{35}$, highlighting the possibility of generating and reprogramming entire a-carboxysome shells.

The $\alpha$-carboxysome proteins of the chemoautotrophic bacterium Halothiobacillus neapolitanus are mostly encoded by genes that are located in a single cso operon, including $c b b L$ and $c b b S$ that encode the large and small subunits of Rubisco, respectively, and genes that encode shell proteins (Fig. 1b). In addition, csoS1D is $\sim 11 \mathrm{kbp}$ downstream of the cso operon and encodes pesudohexameric shell proteins that are most likely responsible for controlling passage of metabolite molecules ${ }^{36,37}$. We generated the synthetic $c s o-1$ and $c s o-2$ operons, which were modified based on the native $H$. neapolitanus cso operon, to heterologously express $\alpha$-carboxysome shell proteins in E. coli BL21(DE3) (Fig. 1b). The cso-1 operon contains the genes encoding carboxysome shell proteins (csoS2, csoS $4 A B, \operatorname{cso} 1 C A B, \operatorname{cso} 1 D)$, as well as $\operatorname{csoSCA}$ that encodes $\beta$-carbonic anhydrase $(\mathrm{CA})^{38}$. The cso-2 operon comprises only the shell protein-encoding genes $(\operatorname{csoS} 2, \operatorname{cso} 4 A B, \operatorname{csoS} 1 C A B$, and $\operatorname{csoS} 1 D)$, without csoSCA. Expression of the $c s o-1$ and $c s o-2$ operons both led to production of higher-ordered shell architectures in the E. coli hosts, as exhibited by thin-section electron microscopy (EM) (Fig. 1c). Sucrose gradient ultracentrifugation and SDS-polyacrylamide gel electrophoresis (SDS-PAGE) indicated the presence of major shell proteins consisting of CsoS1C, CsoS1A, CsoS1B, and CsoS2 (exists as two isoforms CsoS2A and CsoS2B, see detailed description below) enriched in the $20 \%$ sucrose fraction (Fig. 1d). Mass spectrometry further showed the presence of all seven shell proteins and CA encoded by the cso- 1 operon and seven shell proteins encoded by the $c s 0-2$ operon in the $20 \%$ sucrose fraction (Supplementary Tables 1 and 2), confirming the self-assembly of expressed carboxysome shell proteins to form shell supramolecular structures. 

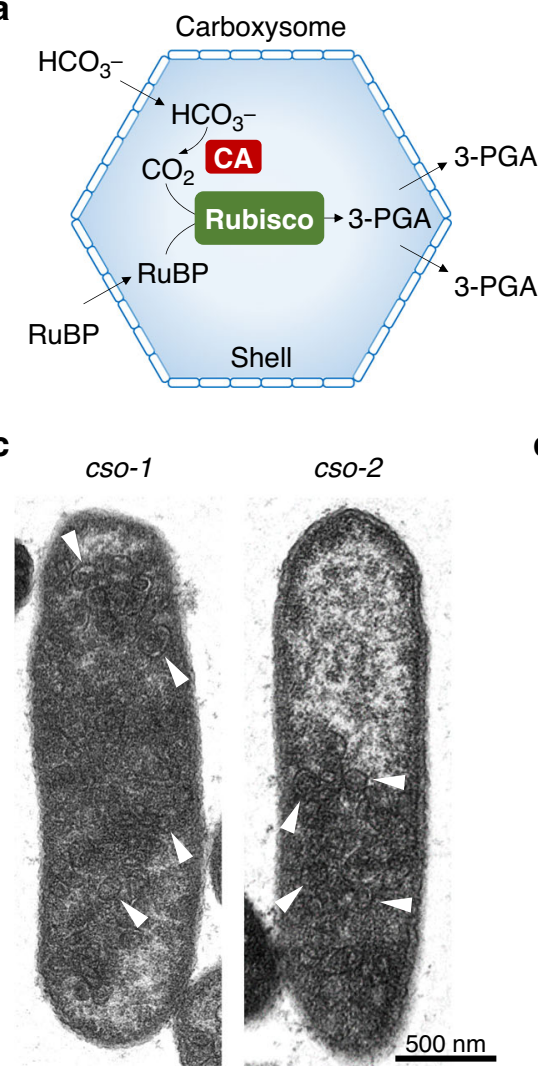

b

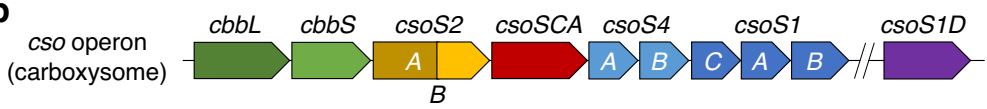

cso-1 operon

$($ shell $+\mathrm{CA})$

cso-2 operon

(shell)

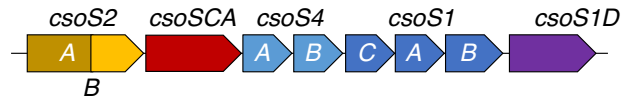

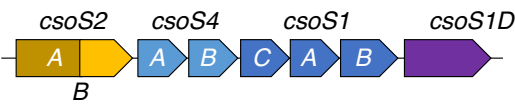

e

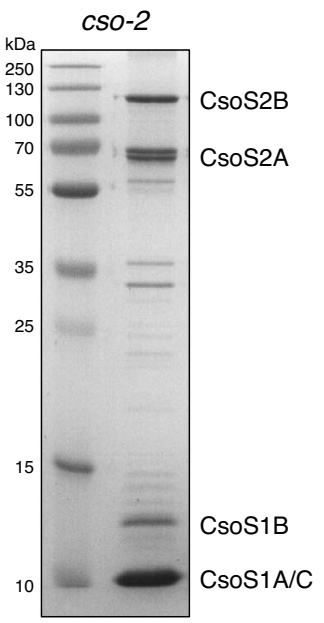

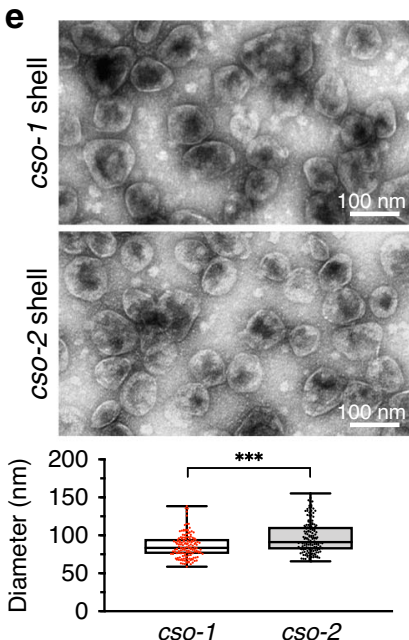

Fig. 1 Engineering and characterization of empty $\boldsymbol{\alpha}$-carboxysome shells. a Schematic of the carboxysome structure and metabolic pathways. The carboxysome shell serves as a physical barrier to permit passage of cytosolic bicarbonate $\left(\mathrm{HCO}_{3}^{-}\right)$and ribulose-1,5-bisphosphate (RuBP) into the carboxysome. The metabolic product 3-phosphoglycerate (3-PGA) is transported across the shell and is metabolized via the Calvin-Benson-Bassham cycle. b Genetic organizations of the native cso operon, synthetic cso-1 and cso-2 operons. c Thin-section electron microscopy (EM) of E. coli cells expressing the cso-1 operon (left) and cso-2 operon (right), respectively. Arrows indicate $\alpha$-carboxysome shell particles discerned in the cell. $\mathbf{d}$ SDS-PAGE of purified cso-1 (left) and cso-2 (right) shell structures. e Transmission EM of purified cso- 1 (top) and cso-2 (middle) shells in the $20 \%$ sucrose fractions. Shells with CA are significantly smaller in diameter than those without CA. ${ }^{\star \star \star} p<0.001$ ( $p=0.0007, n=150$, two-tailed unpaired $t$-test), implying the role of CA in confining shell architecture (bottom). Box and whisker plots indicate the median (middle line in the box), 25th percentile (bottom line of the box), 75th percentile (top line of the box), as well as the minima and maxima (whiskers). Source data underlying c-e are provided as a Source data file.

Negative-staining EM of the 20\% sucrose fraction shows that the recombinant $\alpha$-carboxysome shells exhibit a polyhedral shape, with a diameter of $80-110 \mathrm{~nm}$ (cso-1 shell: $85.93 \pm 15.74 \mathrm{~nm}$; cso-2 shell: $97.12 \pm 20.84 \mathrm{~nm} ; n=150$ ) (Fig. 1e), resembling the native a-carboxysomes from $H$. neapolitanus ${ }^{39}$. This shows that empty a-carboxysome shells can be constructed in the non-native host E. coli, by expressing only shell proteins without cargo proteins, consistent with in vivo observations ${ }^{35}$. This in turn implies the specific assembly pathway of $\alpha$-carboxysomes, either 'shell first' or 'concomitant shell-core assembly', is distinct from the 'inside-out' mode of $\beta$-carboxysome biogenesis ${ }^{32,33}$.

Without cargos, the shells in the same sucrose fraction exhibit variable structures (Fig. 1e). Likewise, the shell size varies among shell structures collected from different sucrose fractions (Supplementary Fig. 1a, b). Dynamic Light Scattering (DLS) analysis revealed that the shell size gradually increases from 10 to $30 \%$ sucrose density (cso- 1 shell: from $82.8 \mathrm{~nm}$ to $104.3 \mathrm{~nm}$; cso-2 shell: from $82.1 \mathrm{~nm}$ to $147.8 \mathrm{~nm}$ ), in agreement with EM results (Supplementary Fig. 1c).

Our results also showed that stable polyhedral shells can be formed in the absence of CA, implicating that CA is not an essential component for shell assembly. This is consistent with previous results, which illustrated that $\alpha$-carboxysomes can be assembled in $H$. neapolitanus without $\mathrm{CA}^{25}$. Moreover, the shells lacking $\mathrm{CA}$ are on average larger than their counterparts containing CA from individual sucrose fractions (Fig. 1e and Supplementary Fig. 1), suggesting that CA plays a role in defining the shell architecture, likely by tight association with shell proteins ${ }^{40,41}$. The effect of CA on shaping the shell structure is restricted in the shells that possess the minimal size $(\sim 82 \mathrm{~nm}, 10 \%$ sucrose, Supplementary Fig. 1b).

CsoS2 is vital for the formation of empty a-carboxysome shells. In the native $a$-carboxysome, CsoS2 is an intriguing disordered protein and has a relatively high abundance. It exists as two distinct isoforms in $H$. neapolitanus: the longer form CsoS2A $(\sim 130 \mathrm{kDa})$ and the short form CsoS2B $(\sim 85 \mathrm{kDa})^{42}$. CsoS2 interacts with shell proteins and its $\mathrm{N}$-terminus is crucial for organising Rubisco inside the $\alpha$-carboxysome $e^{43,44}$, suggesting the important role of CsoS2 in carboxysome assembly ${ }^{45}$, which is functionally analogous to $\mathrm{CcmM}$, the linking proteins of $\beta$ carboxysomes for bridging cargo and the shell ${ }^{31,46}$. To verify the necessity of CsoS2 in the assembly of empty a-carboxysome shells, a cso-3 operon was generated by deleting $c s o S 2$ from the cso-2 operon (Fig. 2a). Thin-section EM showed that no shell structures were discerned in the cso-3 E. coli cells, in contrast to the EM results of the cso-2 cells (Fig. 2b). Non-assembled shell proteins were prone to form aggregates at the pole of the $c s o-3$ cell (Fig. 2b, orange arrow). SDS-PAGE analysis of the pellets of cell 
a

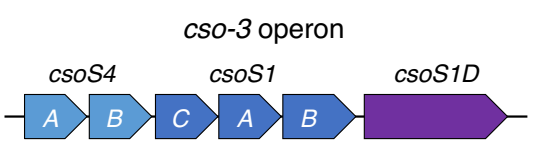

b

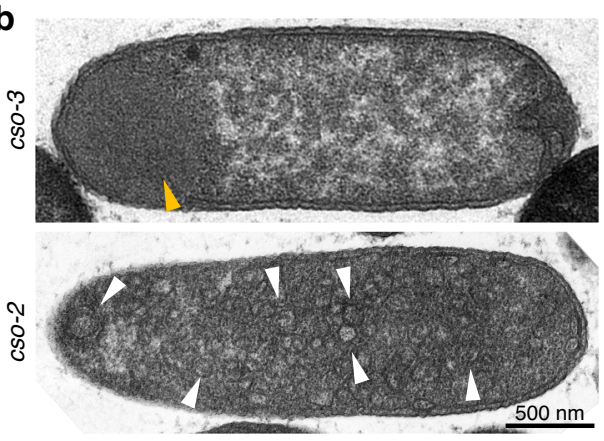

c

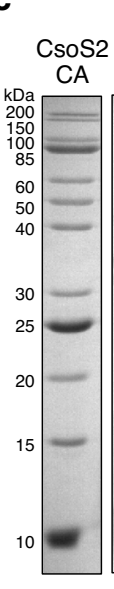

$\begin{array}{llll}C & 1 & 2 & 3 \\ - & + & + & -\end{array}$

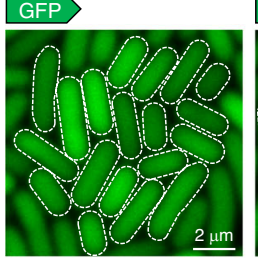

cso-2

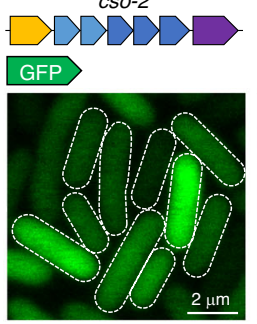

cso-2

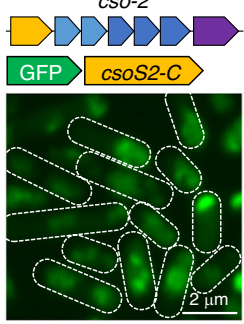

$\mathbf{f}$

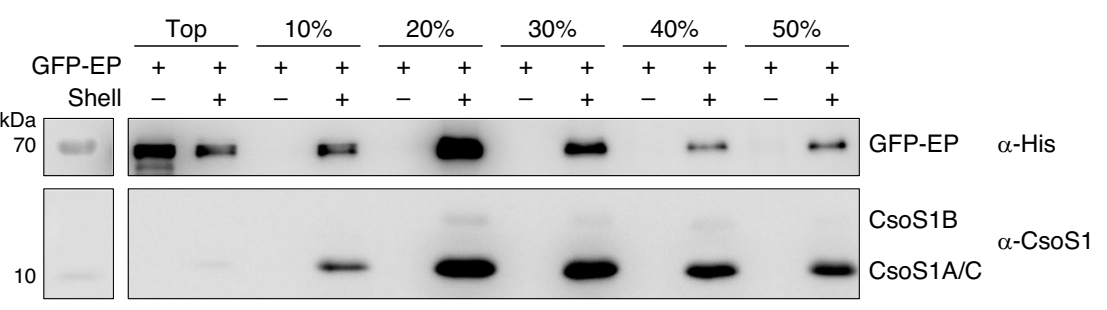

d

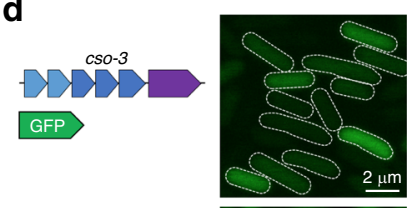

Csos2A

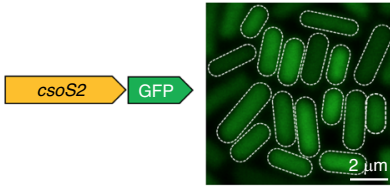

Csos1B

Csos1A/C

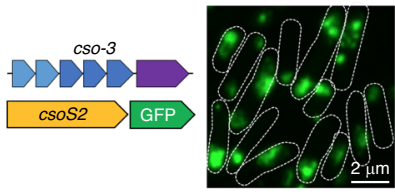

g
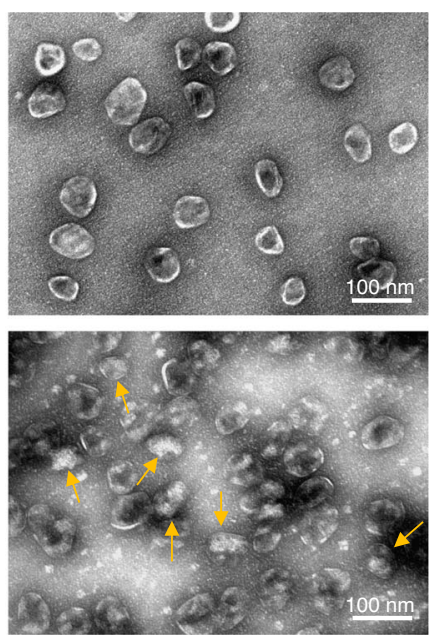

Fig. 2 Roles of CsoS2 in carboxysome shell assembly and identification of CsoS2 C-terminus as the encapsulation peptide. a Genetic organization of the synthetic cso-3 operon. $\mathbf{b}$ Thin-section EM of $E$. coli cells expressing the cso-3 operon (top) and cso-2 operon (bottom). Yellow arrows indicate protein aggregates formed by non-assembled shell proteins in the cso-3 cells. White arrows indicate individual $\alpha$-carboxysome shell particles in the cso- 2 cells. c SDS-PAGE of the $50,000 \times g$ pellets purified from E. coli cells expressing empty plasmid (control, C), cso-1 (1), cso-2 (2) and cso-3 (3) operon, respectively. d Confocal images revealed the formation of shell assemblies in $E$. coli cells in the presence of both CsoS2 and shell proteins. e Confocal images of E. coli cells expressing GFP, GFP-CsoS2 C-terminus (csoS2-C), co-expressing cso-2 and GFP, or co-expressing cso-2 and GFP-csoS2-C, indicating that the CsoS2 C-terminus could function as an encapsulating peptide to mediate incorporation of external proteins into the formed shell structures. f Immunoblot confirms the presence of GFP and shell proteins in the samples purified by sucrose gradient centrifugation from $E$. coli cells producing GFP-EP alone or GFP-EP with shells, using the anti-Histidine (top) and anti-CsoS1A/B/C (below) antibodies (See SDS-PAGE in Supplementary Fig. 3). $\mathbf{g}$ Transmission EM of empty $\alpha$-carboxysome shells (top) and shells with GFP interiors (below) from the $20 \%$ sucrose fractions. Yellow arrows indicate GFP proteins seen in the lumen of the shells. Source data underlying $\mathbf{b}-\mathbf{g}$ are provided as a Source data file.

extracts after $50,000 \times g$ centrifugation showed that shell structures were formed only in the presence of CsoS2 (as indicated by the presence of shell proteins CsoS1A/C and CsoS1B) (Fig. 2c). To further examine the CsoS2-mediated formation of $\alpha$ carboxysome shells, enhanced green fluorescence protein (GFP) was fused to the C-terminus of full-length CsoS2. Without CsoS2 or shell proteins, GFP signal was evenly distributed throughout the cytosol of E. coli (Fig. 2d, top and middle). When CsoS2-GFP and cso-3 shells were co-expressed, spotty fluorescence signal was visualized in the cell's cytosol (Fig. 2d, bottom), signifying the formation of highly ordered shell structures. Collectively, our results revealed that CsoS2 is essential for the formation of empty a-carboxysome shells.

CsoS2 C-terminus acts as the encapsulation peptide to incorporate cargo into the shell. Given the necessity of the intact
$\mathrm{CsoS} 2$ and its C-terminus in the formation of intact carboxysomes ${ }^{43-45}$ and empty shells (Fig. 2), we speculate that the Cterminal fragment of CsoS2 (278 amino acids) links the shell and cargo assemblies, and thereby, may serve as the EP to direct cargo enzymes into the $\alpha$-carboxysome shell (Supplementary Fig. 2). To verify this hypothesis, GFP was fused to the $\mathrm{N}$-terminus of the CsoS2 C-terminal fragment and co-expressed with the cso-2 shells. Confocal images showed that the cells expressing only GFP, GFP-EP, or cso-2 shells and GFP presented diffuse fluorescence signal throughout the cell, whereas the cells expressing cso-2 shells and GFP-EP exhibited explicitly dispersed fluorescent foci (Fig. 2e), demonstrating the EP-mediated cargo encapsulation into the synthetic shell. Recombinant cso-2 shells with incorporated GFP-EP were purified from E. coli by sucrose gradient ultracentrifugation. Immunoblot analysis revealed that the shells encapsulating GFP-EP were detected in the $10-50 \%$ sucrose fractions, with the strongest GFP signal observed in the $20 \%$ 
sucrose fraction (Fig. $2 \mathrm{f}$ and Supplementary Fig. 3). Without shells, by contrast, free GFP-EP were only detected at the top of the sucrose gradient (Fig. 2f), consistent with the quantification of GFP fluorescence in these sucrose fractions (Supplementary Fig. 4). EM of the $20 \%$ sucrose fraction revealed the incorporation of GFP-EP in the shell interior, and that the size of the GFPencapsulated shells is relatively comparable to that of the empty shells (Fig. 2g). These results demonstrate that the C-terminal region of CsoS2 directly interacts with the shell, consistent with the previous finding ${ }^{43}$, and this peptide could serve as an EP to recruit external proteins into the empty shell.

The C-terminal region of CsoS2 has three repeats $\left(\mathrm{R}_{1}-\mathrm{R}_{3}\right)$ and a conserved C-terminal peptide (Supplementary Fig. 2a) ${ }^{45}$. To determine the roles of these domains in cargo encapsulation, we systematically generated the GFP-tagged CsoS2 C-terminus variants that differ in the number of the repeats and the Cterminal peptide. Coexpressing these GFP-CsoS2 C-terminus peptides with the $c s o-2$ shells and confocal images showed that the punctate fluorescence signal relative to the cytoplasmic fluorescence reduced along with the decrease in the numbers of the C-terminal repeats (Supplementary Fig. 5). The C-terminal peptide alone was incapable of mediating incorporation of GFP into the shell. These results indicate that the $R_{1}-R_{3}$ repeats of the CsoS2 C-terminal region are important for cargo encapsulation, probably by interacting with shell proteins; more repeats ensure higher efficiency of cargo encapsulation. Additionally, removal of the C-terminal peptide from the CsoS2 C-terminus (only $\mathrm{R}_{1} \mathrm{R}_{2} \mathrm{R}_{3}$ ) has no significant effects on shell formation and GFP incorporation, supporting the assumption that the C-terminal peptide may be exposed on the outside of the carboxysome shell ${ }^{43}$ (Supplementary Fig. 2d).

Incorporation of [ $\mathrm{FeFe}]$-hydrogenase into the carboxysome shell to create a catalyst for hydrogen production. Though catalytically efficient for hydrogen evolution, [FeFe]-hydrogenases are highly sensitive to $\mathrm{O}_{2}$, representing a barrier for biological $\mathrm{H}_{2}$ production. The potential $\mathrm{O}_{2}$-limited microenvironment in the carboxysome shell is ideal for enhancing the catalytic activities of oxygen-sensitive enzymes. Defining the role of the CsoS2 C-terminus as the EP paves the way for generating a nanoreactor based on the intact a-carboxysome shell, which is capable of incorporating heterologous enzymes for new catalytic functions.

The $[\mathrm{FeFe}]$-hydrogenase from the green alga Chlamydomonas reinhardtii, $\mathrm{HydA}$, is one of the simplest $[\mathrm{FeFe}]$-hydrogenases and represents the 'minimal unit' for biological $\mathrm{H}_{2}$ production ${ }^{47,48}$. We generated a hyd plasmid to express the following proteins: (1) algal $\mathrm{HydA}$ that are fused with algal $\mathrm{Fd}$ at the $\mathrm{N}$-terminus, with a 15-aa linker ${ }^{49}$, and the $\mathrm{EP}$ at the $\mathrm{C}$-terminus. Fd serves as the native electron donor of algal $\mathrm{HydA}$, and the Fd-HydA fusion increased the rate of $\mathrm{H}_{2}$ production ${ }^{49}$; (2) E. coli FNR fused with $\mathrm{EP}$ at the C-terminus. FNR originally from E. coli could catalyse the transfer of electrons from NADPH to $\mathrm{Fd}^{50}$; (3) the maturase enzymes HydE, HydF, and HydG (Fig. 3a), which are crucial for the formation and activation of $\mathrm{HydA}^{51,52}$. In addition, polyhistidine tags (His-tags) were linked to the $\mathrm{N}$-terminus of Fd-HydA and the C-terminus of FNR to facilitate biochemical identification. This plasmid and cso-2 were both transformed into E. coli (Fig. 3a). Expression of the hyd plasmid was induced by addition of isopropyl $\beta$-D-1-thiogalactopyranoside (IPTG) for $4 \mathrm{~h}$ before the expression of the shell induced by $\mathrm{L}$-arabinose. This temporally separated expression strategy ensures maturation and activation of hydrogenases prior to shell formation and encapsulation ${ }^{11}$. During shell assembly, EP fusion to both $\mathrm{Fd}-$ HydA and FNR directs packaging of Fd, HydA and FNR into the shell interior, allowing for installation of a functional electron transfer pathway into the $\alpha$-carboxysome shell and generation of a hydrogenase-containing nanoreactor (Shell-HydA) for consecutive $\mathrm{H}_{2}$ production using electrons from NADPH (Fig. 3b).

After 50,000 $\times g$ centrifugation, the components of Shell-HydA including shell proteins and HydA were detected in the pellet, whereas unencapsulated HydA were only present in the supernatant (Supplementary Fig. 6), implicating the formation of ShellHydA assemblies. Similar to the shells containing GFP-EP (Fig. 2f), Shell-HydA were predominantly enriched in the $20 \%$ sucrose fraction, whereas expressed HydA in the absence of shells were not detectable in the 10-50\% sucrose fractions (Fig. 3c). EM of the Shell-HydA assemblies in the $20 \%$ sucrose fraction further suggested the incorporation of HydA into recombinant carboxysome shells mediated by the EP (Fig. 3d, orange arrows).

The $\mathrm{H}_{2}$ production activity of the generated $E$. coli cells expressing Shell-HydA and free HydA were assayed using endogenous NADPH in the cells as the electron source. Cells were cultivated and induced for $16 \mathrm{~h}$ in either aerobic or anaerobic conditions. The dissolved oxygen (DO) levels of the liquid cultures of two cell types were monitored (Supplementary Fig. 7) and the produced $\mathrm{H}_{2}$ was measured by gas chromatography (Fig. 3e). Although both cell types consumed a large amount of $\mathrm{O}_{2}$ after 16h incubation (DO dropped from $25 \%$ to $1 \%$ for free HydA and from $29 \%$ to $1.3 \%$ for Shell-HydA), the final DO levels of both cell types under aerobic conditions were notably higher than those kept constant under anaerobic conditions $(\sim 0.2 \%)$ (Supplementary Fig. 7a). Under anoxic conditions, the $\mathrm{H}_{2}$-evolution rate of cells expressing Shell-HydA is $4,464.69 \pm 165.49 \mathrm{nmol} \mathrm{L}^{-1} \mathrm{~h}^{-1}$ (mean \pm standard deviation (s.d.), $n=3$ ), which is $\sim 20 \%$ greater than that of cells expressing free HydA $\left(3,729.22 \pm 372.00 \mathrm{nmol} \mathrm{L}^{-1} \mathrm{~h}^{-1}\right)$ (Fig. 3e), suggesting that enzyme catalysis can be more efficient in the closed microcompartment likely due to the increased local HydA concentration and more static interaction with substrates. The discrepancy in $\mathrm{H}_{2}$ evolution between the E. coli hosts producing Shell-HydA and free HydA is explicitly more pronounced under aerobic conditions. The $\mathrm{H}_{2}$-evolution rate of cells producing Shell-HydA $\left(882.95 \pm 154.71 \mathrm{nmol} \mathrm{L}^{-1} \mathrm{~h}^{-1}\right)$ is 4.1-fold greater than that of cells expressing free HydA (217.61 \pm $25.58 \mathrm{nmol} \mathrm{L}^{-1} \mathrm{~h}^{-1}$ ) (Fig. 3e). In light of the extreme oxygen sensitivity of [FeFe]-hydrogenases, the significant improvement of the $\mathrm{H}_{2}$-producing capacity of Shell-HydA compared to that of free HydA under aerobic condition is mainly ascribed to the lower $\mathrm{O}_{2}$ level created within the shell lumen. The possibility that the different hydrogenase activities of the two cell types were ascribed to their distinct oxygen-consumption capacities can be excluded, since no drastic difference in oxygen consumption between the two cell types was determined (Supplementary Fig. 7b). In addition, Shell-HydA cells exhibited a lower $\mathrm{H}_{2}$ yield under aerobic conditions than anaerobic conditions, possibly due to the inactivation of $\mathrm{O}_{2}$ during the maturation and activation processes of [FeFe]-hydrogenases prior to encapsulation by the shells (Fig. 3e). No notable difference in the $\mathrm{H}_{2}$ production activity between HydA encapsulated inside the shell with CA and without $\mathrm{CA}$, under either anaerobic or aerobic conditions was observed (Supplementary Fig. 8).

The Shell-HydA catalysts were further anaerobically purified for in vitro activity assays using methyl viologen (MV) as an electron donor, which were chemically reduced by sodium dithionite. In the assays, the HydA content was measured using purified HydA as the reference (Supplementary Fig. 9). The maximum hydrogen evolution rate of Shell-HydA is $603.33 \pm$ $44.87 \mathrm{nmol} \mathrm{mg}^{-1} \mathrm{~min}^{-1}$ at $\mathrm{pH} 8, \sim 5.5$-fold greater than that of free HydA $\left(109.32 \pm 29.28 \mathrm{nmol} \mathrm{mg}^{-1} \mathrm{~min}^{-1}\right)$ (Fig. 4a), comparable with the results of in vivo activity assays (Fig. 3e). The amount of hydrogen produced by Shell-HydA measured at 
a

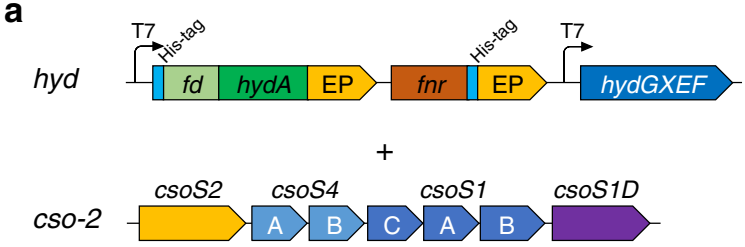

C Cd-HydA-EP $^{\mathrm{CE}} \frac{10 \%}{+++} \frac{20 \%}{++} \frac{30 \%}{++++} \frac{40 \%}{+++}$

$\mathrm{KDa}$ Shell -+-++-++-+

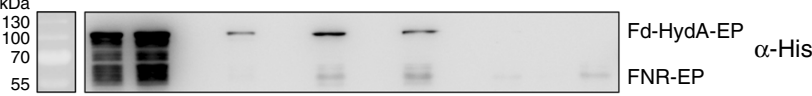

$\sqrt[15]{\square-0}$ Csos1B

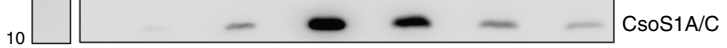

d
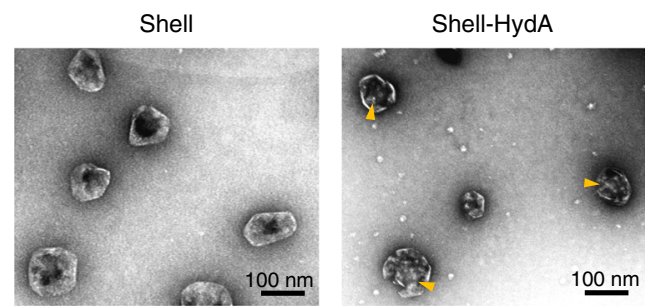

b

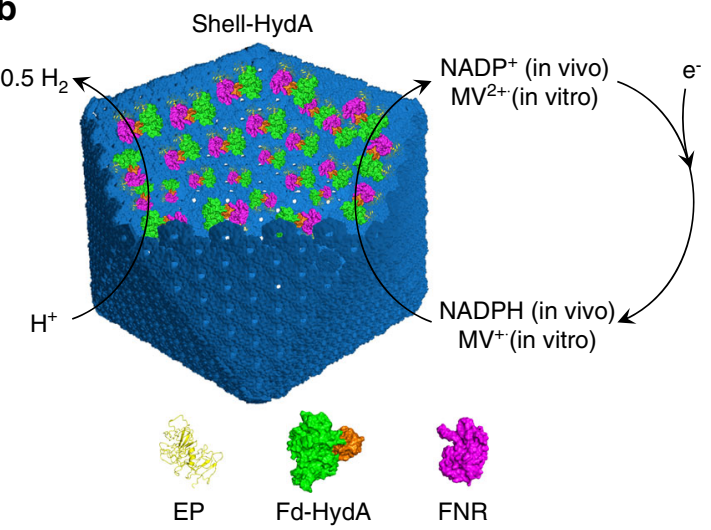

$\alpha-\operatorname{CsoS1} \mathbf{e}$

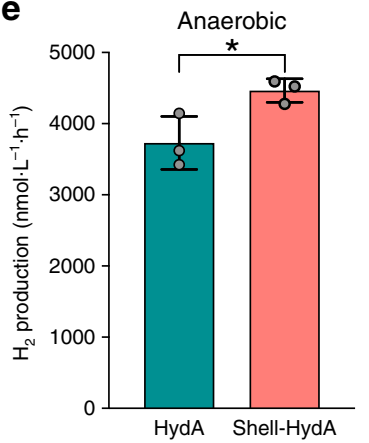

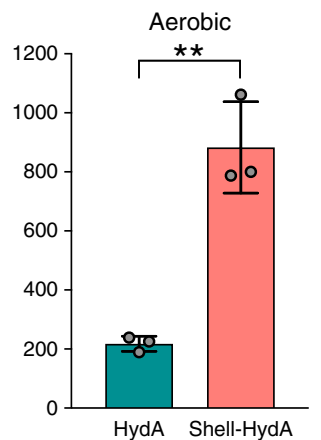

Fig. 3 Construction of a hydrogenase-containing nanoreactor based on the carboxysome shell. a Generation of the hyd operon to produce [FeFe]hydrogenase HydA fused with ferredoxin (Fd) from the green alga Chlamydomonas, ferredoxin:NADP+-oxidoreductase (FNR) from E. coli, and the HydA maturases HydE, HydF, and HydG. The hyd and cso-2 plasmids were co-expressed to form a hydrogenase-containing nanoreactor based on the carboxysome shell (Shell-HydA). b Schematic of the Shell-HydA nanoreactor encapsulating Fd-HydA and FNR. The nanoreactors were tested for hydrogen production activity using endogenous NADPH in cells as the electron donor for in vivo assays, and for in vitro assays, using methyl viologen (MV ${ }^{+}$) as an electron donor, which were chemically reduced by sodium dithionite. c Western blot of E. coli expressing the hyd operon alone or Shell-HydA confirms the presence of Fd-HydA, FNR, and shell proteins in the samples purified from sucrose gradient ultracentrifugation, indicating the assembly of Shell-HydA. d Transmission EM of empty shells (left) and Shell-HydA (right). Yellow arrows indicate cargo proteins (Fd-HydA, FNR) in the luminal side of the shell. e In vivo hydrogenase activity assays. $\mathrm{H}_{2}$ production $\left(\mathrm{nmol} \mathrm{L} \mathrm{L}^{-1} \mathrm{~h}^{-1}\right.$ ) of $E$. coli cells expressing free HydA or Shell-HydA grown under anaerobic (left) or aerobic (right) conditions was measured using gas chromatography. Values represent mean \pm standard deviations (s.d.), $n=3$ biologically independent experiments. ${ }^{\star} p=0.0352$ (left), ${ }^{\star \star} p=0.0018$ (right) (two-tailed unpaired $t$-test). Source data underlying c-e are provided as a Source data file.
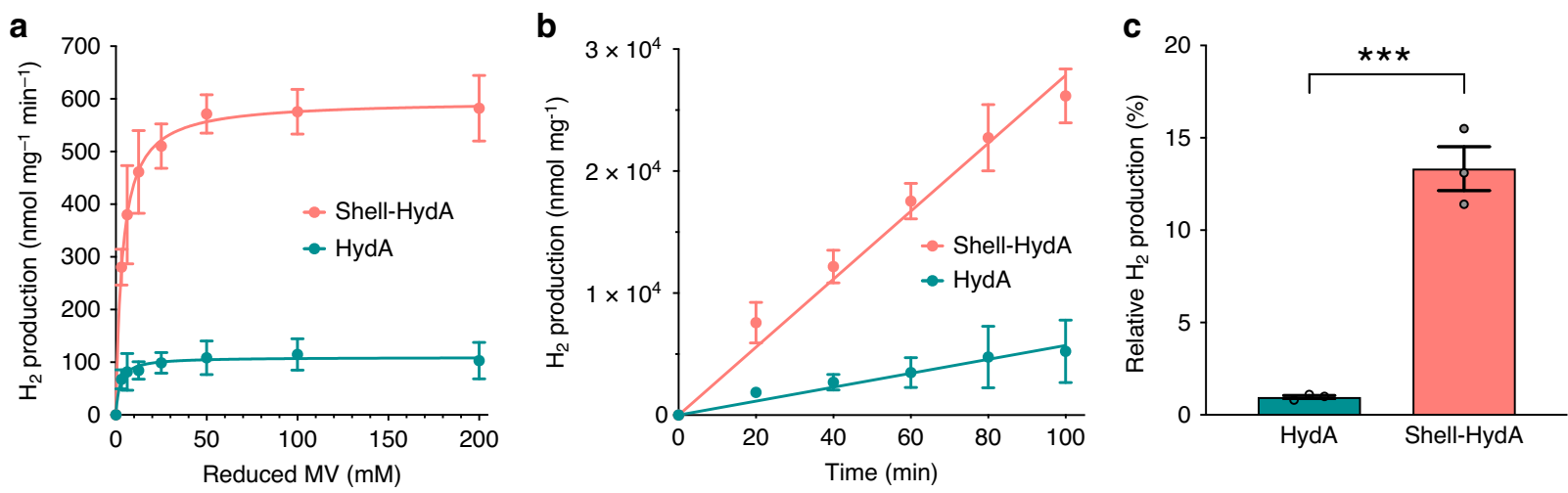

Fig. 4 In vitro Hydrogen production of the carboxysome shell-based nanoreactor. a Hydrogen production activity $\left(\mathrm{nmol} \mathrm{mg}^{-1} \mathrm{~min}^{-1}\right)$ of isolated free HydA and Shell-HydA at pH 8 as a function of different concentrations of $\mathrm{MV}^{+}$as the electron mediator reduced by sodium dithionite (DT), fitted with Michaelis-Menten kinetics. Values represent mean \pm s.d., $n=3$ biologically independent experiments. $\mathbf{b}$ Kinetic hydrogen production of free HydA and Shell-HydA using $50 \mathrm{mM}$ DT reduced $\mathrm{MV}^{+}$as the electron mediator at $\mathrm{pH}$ 8. Values represent mean \pm s.d., $n=3$ biologically independent experiments. c Relative activity of free HydA and Shell-HydA after oxygen exposure for $24 \mathrm{~h}$ at $4{ }^{\circ} \mathrm{C}$, as a relative percentage of total activities measured under anaerobic conditions (see also Supplementary Fig. 10). Values represent mean \pm s.d., $n=3$ biologically independent experiments. ${ }^{\star \star *} p=0.0009$ (two-tailed unpaired $t$-test). Source data are provided as a Source data file. 
$50 \mathrm{mM} \mathrm{MV}$ increases linearly over time, indicating that the process is indeed catalytic (Fig. 4b). Moreover, Shell-HydA produces remarkably more hydrogen under these conditions than free HydA, emphasising the advantage of the carboxysome shellorganized nanoreactor in enhancing the $\mathrm{H}_{2}$ production activity of encapsulated hydrogenases.

To further investigate the $\mathrm{O}_{2}$ tolerance of the shell-based nanoreactor, the Shell-HydA catalysts purified under anaerobic conditions were exposed to the air for $24 \mathrm{~h}$ followed by activity assays. After oxygen exposure, Shell-HydA retained $13.33 \pm 2.06 \%$ activity relative to the activity measured under anaerobic conditions, whereas only $0.97 \pm 0.15 \%$ activity was maintained for free HydA exposed to $\mathrm{O}_{2}$ (Fig. $4 \mathrm{c}$ and Supplementary Fig. 10), indicating greater oxygen tolerance of HydA encased inside the carboxysome shell compared to free HydA.

Overall, our results demonstrated that the Shell-HydA nanoreactors are catalytically active for $\mathrm{H}_{2}$ production, and encapsulation of the carboxysome shell is beneficial to the $\mathrm{H}_{2}$ production activity and oxygen tolerance of encased hydrogenases. Several possibilities could result in the enhancement of catalytic performance of [ $\mathrm{FeFe}]$-hydrogenases encapsulated by the carboxysome shell. One possibility is that the selectively permeable shell may create a 'low- $\mathrm{O}_{2}$ microenvironment' around $[\mathrm{FeFe}]$-hydrogenases by preventing the entry of $\mathrm{O}_{2}{ }^{25,53}$, thereby promoting the $\mathrm{H}_{2}$-production activities of highly oxygen-sensitive [FeFe]-hydrogenases. Shell encapsulation could also allow for condensation of enzymes and more static interaction with substrates within the microcompartment (as indicated by a $20 \%$ increase in $\mathrm{H}_{2}$ production of Shell-HydA under anaerobic conditions compared to that of free HydA, Fig. 3c), which could facilitate enzyme catalysis ${ }^{54,55}$. Moreover, the protein shell may confer physical protection to its cargo encapsulated from proteolytic cleavage ${ }^{11}$.

It was proposed that the carboxysome shell allows passage of negatively charged metabolites ${ }^{53,56,57}$ and protons ${ }^{58}$ across the shell. The main shell proteins in the $\alpha$-carboxysome, CsoS1A hexamers, contain a narrow pore with a diameter of $\sim 4 \AA^{56}$, whereas the CsoS1D pseudohexamers have a larger pore with a diameter ranging between 0 and $18 \AA^{37}$. The interior of this channel is mainly positively charged, paving the way for passage of large negatively charged metabolites (such as NADPH) across the shell. The $\mathrm{H}_{2}$ production experiments suggested that the positive protons and negative NADPH, as well as the catalytic product $\mathrm{H}_{2}$, can diffuse across the shell (Fig. 3b). Precisely how the carboxysome shell permits diffusion of small $\mathrm{H}_{2}$ molecules merits further investigation.

In summary, we generated and characterized large and robust carboxysome shells with a diameter of $\sim 100 \mathrm{~nm}$. We also demonstrated the feasibility of reprograming the synthetic protein cages, by sequestering a catalytically active, $\mathrm{H}_{2}$ producing pathway within the shell, to boost production of hydrogen. The heterologously engineered shells possess similar protein composition and architectures relative to the carboxysome shells from the native hosts, suggesting the great capacity of cargo loading. We provide evidence that CsoS2 is essential for the assembly of the empty shell, and the C-terminus of CsoS2 could serve as an EP for recruiting external cargo proteins into the synthetic shells. Taking advantage of the defined EP and self-assembly of the carboxysome shells, we installed active [FeFe]-hydrogenases and Fd from green algae together with $E$. coli FNR inside the empty shell to create a large proteinaceous nanoreactor for hydrogen production. One limitation of using endogenous C-terminus of CsoS2 as EP may be the relatively low loading capacity of cargo enzymes, given the limited interaction site of CsoS2 on the shell. Developing efficient encapsulation strategies to improve cargo loading and protein organization is required in future explorations. The shell encapsulation and the specific microenvironment created in the engineering nanoreactor were demonstrated to favour the catalytic activity of oxygen-sensitive [FeFe]-hydrogenases and improve $\mathrm{O}_{2}$ tolerance of the generated catalysts significantly. Advanced understanding of the assembly principles of carboxysomes and shells, and the developed engineering systems for precisely tuning enzyme activation, shell formation and encapsulation will inform rational design and engineering of carboxysome shell-based nanomaterials and nanostructures in biotechnological applications for catalytic enhancement, enzyme protection, and molecule delivery. We also see potential to combine these biological assemblies with abiotic cocatalysts in the future.

\section{Methods}

Generation of constructs. All connections between genes and linearized vectors were achieved by Gibson assembly (Gibson assembly kit, New England BioLabs, $\mathrm{UK})$. The nucleotide sequence of the a-carboxysome shell operon encoding CsoS2, CsoSCA, CsoS4A, CsoS4B, CsoS1C, CsoS1A, CsoS1B, and CsoS1D was amplified from the genome of Halothiobacillus neapolitanus and was cloned into the pBAD vector linearized by $\mathrm{NcoI}$ and EcoRI (cso- 1 vector). The $\operatorname{csoS} 2$ gene and the nucleotide sequence encoding CsoS4A, CsoS4B, CsoS1C, CsoS1A, CsoS1B, and CsoS1D were amplified from $c s o-1$ and assembled into $\mathrm{pBAD}$ vector linearized by $\mathrm{NcoI}$ and EcoRI (cso-2 vector). The genes encoding CsoS4A, CsoS4B, CsoS1C, CsoS1A, CsoS1B, and CsoS1D were amplified from the cso-1 vector and inserted into pBAD vector linearized by $\mathrm{NcoI}$ and EcoRI to generate the cso- 3 vector. The nucleotide sequence of the C-terminus of full-length CsoS2 was amplified from the cso- 1 vector. The hydA (GenBank accession code AAL23572.1) and fd (GenBank accession code XP_001692808.1) genes from Chlamydomonas reinhardtii were codon-optimized for heterologous expression in E. coli. The Fd protein was fused to the N-terminus of HydA with a 15 amino acid linker composed of GGGGSGGGGSGGGGS (Supplementary Table 3). The hydGX and hydEF genes from Shewanella oneidensis (GenBank accession code AE014299.2: 40701484074530), which encode the maturases HydE, HydF and HydG were synthesized. The fnr gene (GenBank accession code WP_053887749.1) was amplified from the genomic DNA of E. coli BL21(DE3). The $f d-h y d A$ and fnr genes were separately fused with the nucleotide sequence of EP and then ligated to pCDFDueT-1 linearized by EcoRI and AscI, together with the hydGX and hydEF gene fragments, to generate the hyd vector. The $g f p$ gene was cloned to pCDFDueT-1 linearized by EcoRI and AscI to generate the pCDF- $g f p$ vector. The $g f p$ gene with the nucleotide sequence of EP fused at the C-terminus was cloned into pCDFDueT-1 and in frame with the nucleotide sequence encoding $6 \mathrm{x}$ polyhistidine tag to create $\mathrm{pCDF}-g f p-\mathrm{EP}$ The $g f p$ gene fused to the C-terminal of full-length $\operatorname{csoS} 2$ gene was inserted into pCDFDueT-1 linearized by EcoRI and AscI to construct pCDF-csoS2-gfp. The truncations of the CsoS2 C-terminal region were shown in Supplementary Fig. 5. The genes encoding truncated CsoS2 C-terminals were amplified using PCR from plasmid pCDF- $g f p$-EP. The $g f p$ gene fused to the N-terminal of these truncated CsoS2 C-terminal regions were inserted into pCDFDueT-1 linearized by BamHI and AscI. All of these constructs were verified by PCR and DNA sequencing and transformed into E. coli DH5a and BL21(DE3) cells.

Heterogeneously generation of $\boldsymbol{\alpha}$-carboxysome shells. E. coli strains containing the cso- 1, cso- 2 or $c s o-3$ vectors were cultivated at $37^{\circ} \mathrm{C}$ in Lysogeny Broth (LB) medium containing $100 \mu \mathrm{g} \mathrm{mL}^{-1}$ ampicillin. The expression of these vectors was induced by L-Arabinose $(1 \mathrm{mM}$, final concentration) once the cells reached early log phase $\left(\mathrm{OD}_{600}=0.6\right)$. Cells were grown at $25^{\circ} \mathrm{C}$ for $16 \mathrm{~h}$ with constant shaking and then were harvested by centrifugation at $4000 \times g$ for $10 \mathrm{~min}$. The cell pellets were washed with TEMB buffer $(5 \mathrm{mM}$ Tris- $\mathrm{HCl} \mathrm{pH}=$ 8.0, $1 \mathrm{mM}$ EDTA, $10 \mathrm{mM} \mathrm{MgCl}_{2}, 20 \mathrm{mM} \mathrm{NaHCO}_{3}$ ) and resuspended in TEMB buffer supplemented with $10 \%(\mathrm{v} / \mathrm{v})$ CelLytic B cell Lysis reagent (SigmaAldrich) and $1 \%$ Protein Inhibitor Cocktail (100x) (Sigma-Aldrich). The cell suspensions were lysed by French Press (Stansted Fluid Power, UK). Cell debris was removed by centrifugation, followed by centrifugation at $50,000 \times g$ to enrich a-carboxysome shells. The pellets were resuspended in TEMB buffer and then loaded onto sucrose gradients $(10-30 \%$ or $10-50 \%$, w/v) followed by ultracentrifugation (BeckMan, XL100K Ultracentrifuge) at $105,000 \times g$ for $30 \mathrm{~min}$. Each sucrose fractions were collected and stored at $4{ }^{\circ} \mathrm{C}$.

Recombinantly expression of GFP, GFP-EP and CsoS2-GFP. E. coli strains containing pCDF- $g f p$, pCDF- $g f p-\mathrm{EP}$ or $\mathrm{pCDF}-\mathrm{csoS} 2-g f p$ were grown in LB containing $50 \mu \mathrm{g} \cdot \mathrm{mL}^{-1}$ spectinomycin at $37^{\circ} \mathrm{C}$. The expression of these vectors was induced by $0.5 \mathrm{mM}$ (final concentration) isopropyl $\beta$-D-thiogalactopyranoside (IPTG) once the cells reached early log phase $\left(\mathrm{OD}_{600}=0.6\right)$, followed by cell cultivation for $16 \mathrm{~h}$ at $25^{\circ} \mathrm{C}$. 
Expression of mature [FeFe]-hydrogenase and generation of $\alpha$-carboxysome shells with encapsulated hydrogenases. For the expression of mature, functional [FeFe]-hydrogenases as well as Fd and FNR, E. coli cells containing the hyd vector grown in LB medium containing $0.2 \mathrm{mM}$ ferric ammonium citrate and $50 \mu \mathrm{g} \mathrm{mL}^{-1}$ spectinomycin was induced by the addition of $0.5 \mathrm{mM} \mathrm{IPTG}$, as well as $0.2 \mathrm{mM} \mathrm{L-}$ cysteine and $2.5 \mathrm{mM}$ sodium fumarate (final concentration) at $\mathrm{OD}_{600}=0.7-0.8$. For the co-expression of a-carboxysome shells and mature hydrogenases to produce Shell-HydA, E. coli strains containing the hyd vector and the cso-1 or cso-2 vector were cultivated in LB medium containing $0.2 \mathrm{mM}$ ferric ammonium citrate, $50 \mu \mathrm{gL}^{-1}$ spectinomycin, and $100 \mu \mathrm{g} \mathrm{mL}^{-1}$ ampicillin. The hyd expression was induced by the addition of $0.5 \mathrm{mM}$ IPTG at $\mathrm{OD}_{600}=0.7$. After 4 -h induction of the hyd expression, the shell expression was induced by $1 \mathrm{mM} \mathrm{L}$-Arabinose, and cells were then grown at $25^{\circ} \mathrm{C}$ for $16 \mathrm{~h}$. The hyd induction was performed before the expression of shells to allow hydrogenase maturation prior to shell encapsulation. This temporally separated expression strategy ensures maturation and activation of hydrogenases prior to shell formation and encapsulation ${ }^{11}$. The purification of HydA and Shell-HydA was carried out following the method for shell purification mentioned above.

Hydrogenase activity assay. For in vivo activity assay, E. coli strains were first grown aerobically at $37^{\circ} \mathrm{C}$ until $\mathrm{OD}_{600}$ reached $0.7-0.8$. Cells were then transferred to falcon tubes, sealed with rubber turn-over closures (Sigma-Aldrich), and degassed by $100 \%$ nitrogen for 15 mins before the addition of IPTG, Lcysteine, and sodium fumarate for anaerobic treatment. For aerobic treatment, culture tubes were sealed without nitrogen degassing process. L-Arabinose was added $4 \mathrm{~h}$ after IPTG induction. Then, the cells were grown at $25^{\circ} \mathrm{C}$ for $16 \mathrm{~h}$. Hydrogen produced in the culture tubes was detected by gas chromatography. In all, $1 \mathrm{~mL}$ gas samples were taken with a gas-tight syringe and a sample loop was flushed $(100 \mu \mathrm{L})$ with the sample. The sample loop was then switched and run on a Bruker $450-\mathrm{GC}$ gas chromatograph. The system was equipped with a molecular sieve $13 \times 60-80$ mesh $1.5 \mathrm{~m} \times 1 / 8$ in. $\times 2 \mathrm{~mm}$ ss column at $50^{\circ} \mathrm{C}$ with an argon flow of $40.0 \mathrm{~mL} \mathrm{~min}^{-1}$. Hydrogen was detected by a thermal conductivity detector referencing against standard gas with a known concentration of hydrogen. For each experiment, at least three biological replicates were examined.

For in vitro activity assay, strains were grown aerobically at $37^{\circ} \mathrm{C}$ until $\mathrm{OD}_{600}$ reached $0.7-0.8$, then degassed by $100 \%$ nitrogen before the addition of IPTG, $\mathrm{L}$-cysteine and sodium fumarate. L-Arabinose was added $4 \mathrm{~h}$ after IPTG induction. Cultures were then cultivated at $25^{\circ} \mathrm{C}$ with constant shaking for $16 \mathrm{~h}$. Cell harvesting and protein purification were carried out in an anaerobic chamber (Don Whitley Scientific, MACS-MG-500). The in vitro hydrogen evolution assays were performed inside an anaerobic glove bag (The Versatile AtmosBag, Sigma-Aldrich) flushed with $100 \% \mathrm{~N}_{2}$ before measurements. In vitro activity assay of free HydA was performed as a control for comparison with the hydrogen evolution activity of Shell-HydA.

For in vitro hydrogenase kinetics assays, the protein amount of HydA in the samples containing free HydA or Shell-HydA were quantified by immunoblot using purified HydA as the reference. Then, samples $\left(0.5 \mathrm{~mL}, 10-15 \mathrm{mg} \mathrm{mL}^{-1}\right)$ containing equal amount of HydA in TMB buffer $(5 \mathrm{mM}$ Tris- $\mathrm{HCl} \mathrm{pH} 8.0,10 \mathrm{mM}$ $\mathrm{MgCl}_{2}$ and $20 \mathrm{mM} \mathrm{NaHCO}$ ) were mixed with $100 \%$ nitrogen degassed $\mathrm{MV}$ $(0-200 \mathrm{mM}$, final) and sodium dithionite $(500 \mathrm{mM}$, final) in serum vials (Agilent Technologies) inside the anaerobic glove bag. The vials were incubated at $37^{\circ} \mathrm{C}$ for $100 \mathrm{~min}$ with constant shaking and were then assayed for hydrogen production. Hydrogenase activity at a range of MV concentrations was plotted and fit using a standard Michaelis-Menten model. In addition, hydrogen evolution of free HydA and Shell-HydA at $50 \mathrm{mM}$ MV and $500 \mathrm{mM}$ sodium dithionite was measured every 20 min using gas chromatography. For each experiment, at least three biological replicates were examined.

Oxygen exposure treatment. The HydA and Shell-HydA samples $(0.5 \mathrm{~mL}$, $10-15 \mathrm{mg} \mathrm{mL}^{-1}$ ) used in the in vitro activity assays were exposed to the air for $24 \mathrm{~h}$ at $4{ }^{\circ} \mathrm{C}$ and were then sealed with rubber turn-over closures, followed by $100 \%$ nitrogen degassing. The degassed samples were mixed with MV $(50 \mathrm{mM}$, final $)$ and sodium dithionite $\left(500 \mathrm{mM}\right.$, final). The vials were incubated for $16 \mathrm{~h}$ at $37^{\circ} \mathrm{C}$ with constant shaking and were then assayed for hydrogen production. All buffers used in the experiments were pre-degassed by $100 \%$ nitrogen. For each experiment, at least three biological replicates were examined.

Dissolved oxygen measurement. The dissolved oxygen (DO) levels of strains expressing HydA or Shell-HydA used for in vivo activity assay were monitored by a polarographic DO probe (New BrunswickTM BioFlo/CellGen 115 Fermentor, Eppendorf) during 16-h induction. The polarized polarographic DO probe was calibrated by oxygen saturated water $(100 \% \mathrm{DO})$ and sodium dithionite treated water $(0 \% \mathrm{DO})$, respectively. DO levels of samples were measured before IPTG addition and every four hours after IPTG addition. For each experiment, three biological replicates were examined.

Mass spectrometry. The shell samples collected from sucrose fractions were washed with PBS buffer and were treated for mass spectrometry analysis ${ }^{24}$.
Data-dependent LC-MS/MS analysis was performed on a QExactive quadrupoleOrbitrap mass spectrometer coupled to a Dionex Ultimate 3000 RSLC nano-liquid chromatograph (Thermo Fisher, UK) installed with Xcalibur software version 4.1. A Mascot Generic File, created by Progenesis QI (Version 3.0, Nonlinear Dynamics, Newcastle upon Tyne, UK), was searched against the $H$. neapolitanus carboxysome protein database from UniProt.

SDS-PAGE and immunoblot analysis. SDS-PAGE and immunoblot examination were performed following the procedure described previously ${ }^{59}$. Briefly, $30 \mu \mathrm{g}$ of total protein was loaded into each well. Immunoblot analysis was performed using primary mouse monoclonal anti-His (Invitrogen, dilution 1:3000), rabbit polyclonal anti-CsoS1 (Agrisera, dilution 1:3000), and horseradish peroxidaseconjugated goat anti-mouse IgG secondary antibody (Agrisera, dilution 1:10,000) and anti-rabbit IgG secondary antibody (Agrisera, dilution 1:10,000). Signals were visualized using a chemiluminescence kit (Bio-Rad). Immunoblot images were collected by ImageQuant LAS 4000 software version 1.2.1.119. Immunoblot protein quantification was performed using ImageJ software (version $1.52 \mathrm{~h}$ ). For each experiment, at least three biological repeats were examined.

Dynamic light scattering analysis. In all, $1.5 \mathrm{~mL}\left(20 \mathrm{mg} \mathrm{mL}^{-1}\right.$ total protein) of individual sucrose fractions $(10 \%, 15 \%, 20 \%, 25 \%, 30 \%)$ containing shell particles were analyzed by Dynamic light scattering (DLS ZetaSizer controlled by malvern zetasizer software version 7.1.1) to measure the size distribution and average size of the shells. For each experiment, at least three biological repeats were examined.

Transmission electron microscopy. Thin-section transmission electron microscopy (EM) was performed to visualize the reconstituted shell structures in E. coli strains $s^{21,59,60}$. Isolated shell structures were characterized using negative staining $\mathrm{EM}^{24,29}$. Images were recorded using an FEI Tecnai G2 Spirit BioTWIN transmission electron microscope equipped with a Gatan Rio 16 camera. Image analysis was carried out using ImageJ software (version $1.52 \mathrm{~h}$ ). Statistical analysis was performed using Student's t-test.

Confocal microscopy. Overnight induced $E$. coli cells were immobilized by drying a droplet of cell suspension onto LB agar pads as described before ${ }^{59}$. Blocks of agar with the cells absorbed onto the surface were covered with a coverslip and placed under the microscope. Laser-scanning confocal fluorescence microscopy imaging was performed on a Zeiss LSM780 confocal microscope with a $63 \times / 1.4 \mathrm{NA}$ oilimmersion objective with excitation wavelength at $488 \mathrm{~nm}$ and emission at $520 \mathrm{~nm}$. Live-cell images were recorded from at least five different cultures. All images were captured with all pixels below saturation. Image analysis was carried out using ImageJ software (version $1.52 \mathrm{~h}$ ).

Statistics and reproducibility. All experiments reported here were performed at least three times independently and at least three biological repeats were performed for each experiment.

Reporting summary. Further information on research design is available in the Nature Research Reporting Summary linked to this article.

\section{Data availability}

Data supporting the findings of this work are available within the paper and its Supplementary Information files. A reporting summary for this article is available as a Supplementary Information file. All data are available from the corresponding author upon request. The protein sequence of Fd, HydA and FNR are available in NCBI with the accession code XP_001692808.1, AAL23572.1 and WP_053887749.1, respectively. The protein sequence of HydGXEF is available in NCBI with the accession code AE014299.2 (region 4070148-4074530). The protein sequence in Supplementary Fig. $2 \mathrm{~b}$ is available in NCBI with the accession code WP_081441107.1. Source data are provided with this paper.

Received: 11 April 2020; Accepted: 7 October 2020; Published online: 28 October 2020

\section{References}

1. Esmieu, C., Raleiras, P. \& Berggren, G. From protein engineering to artificial enzymes-biological and biomimetic approaches towards sustainable hydrogen production. Sustain Energy Fuels 2, 724-750 (2018).

2. Rahman M. Z., Kibria M. G. \& Mullins C. B. Metal-free photocatalysts for hydrogen evolution. Chem. Soc. Rev. 49, 1887-1931 (2020).

3. Birrell, J. A., Rudiger, O., Reijerse, E. J. \& Lubitz, W. Semisynthetic hydrogenases propel biological energy research into a new era. Joule 1, 61-76 (2017). 
4. Wang, Q. \& Domen, K. Particulate photocatalysts for light-driven water splitting: mechanisms, challenges, and design strategies. Chem. Rev. 120, 919-985 (2020)

5. Caserta, G., Roy, S., Atta, M., Artero, V. \& Fontecave, M. Artificial hydrogenases: biohybrid and supramolecular systems for catalytic hydrogen production or uptake. Curr. Opin. Chem. Biol. 25, 36-47 (2015).

6. Ogata, H., Lubitz, W. \& Higuchi, Y. Structure and function of $[\mathrm{NiFe}]$ hydrogenases. J. Biochem. 160, 251-258 (2016).

7. Esselborn, J., Kertess, L., Apfel, U.-P., Hofmann, E. \& Happe, T. Loss of specific active-site iron atoms in oxygen-exposed [FeFe]-hydrogenase determined by detailed X-ray structure analyses. J. Am. Chem. Soc. 141, 17721-17728 (2019).

8. Swanson, K. D. et al. [FeFe]-hydrogenase oxygen inactivation is initiated at the $\mathrm{H}$ cluster 2Fe subcluster. J. Am. Chem. Soc. 137, 1809-1816 (2015)

9. Menzel, K. et al. [FeFe]-hydrogenase models assembled into vesicular structures. J. Liposome Res 24, 59-68 (2014).

10. Wilkerson, J. W., Yang, S.-O., Funk, P. J., Stanley, S. K. \& Bundy, B. C. Nanoreactors: Strategies to encapsulate enzyme biocatalysts in virus-like particles. N. Biotechnol. 44, 59-63 (2018).

11. Jordan, P. C. et al. Self-assembling biomolecular catalysts for hydrogen production. Nat. Chem. 8, 179-185 (2016).

12. Bobik, T. A., Lehman, B. P. \& Yeates, T. O. Bacterial microcompartments: widespread prokaryotic organelles for isolation and optimization of metabolic pathways. Mol. Microbiol 98, 193-207 (2015).

13. Tanaka, S., Sawaya, M. R. \& Yeates, T. O. Structure and mechanisms of a protein-based organelle in Escherichia coli. Science 327, 81-84 (2010).

14. Yang, M. et al. Decoding the stoichiometric composition and organisation of bacterial metabolosomes. Nat. Commun. 11, 1976 (2020).

15. Yeates, T. O., Crowley, C. S. \& Tanaka, S. Bacterial microcompartment organelles: protein shell structure and evolution. Annu. Rev. Biophys. 39, 185-205 (2010).

16. Sutter, M., Greber, B., Aussignargues, C. \& Kerfeld, C. A. Assembly principles and structure of a 6.5-MDa bacterial microcompartment shell. Science 356, 1293-1297 (2017).

17. Kerfeld, C. A. \& Erbilgin, O. Bacterial microcompartments and the modular construction of microbial metabolism. Trends Microbiol 23, 22-34 (2015).

18. Kerfeld, C. A., Aussignargues, C., Zarzycki, J., Cai, F. \& Sutter, M. Bacterial microcompartments. Nat. Rev. Microbiol 16, 277-290 (2018).

19. Rae, B. D., Long, B. M., Badger, M. R. \& Price, G. D. Functions, compositions, and evolution of the two types of carboxysomes: polyhedral microcompartments that facilitate $\mathrm{CO}_{2}$ fixation in cyanobacteria and some proteobacteria. Microbiol. Mol. Biol. Rev. 77, 357-379 (2013).

20. Huang, F. et al. Rubisco accumulation factor 1 (Raf1) plays essential roles in mediating Rubisco assembly and carboxysome biogenesis. Proc. Natl Acad. Sci. USA 117, 17418-17428 (2020).

21. Sun, Y., Wollman, A. J. M., Huang, F., Leake, M. C. \& Liu, L. N. Singleorganelle quantification reveals the stoichiometric and structural variability of carboxysomes dependent on the environment. Plant Cell 31, 1648-1664 (2019).

22. Kerfeld, C. A. et al. Protein structures forming the shell of primitive bacterial organelles. Science 309, 936-938 (2005).

23. Tanaka, S. et al. Atomic-level models of the bacterial carboxysome shell. Science 319, 1083-1086 (2008).

24. Faulkner, M. et al. Direct characterization of the native structure and mechanics of cyanobacterial carboxysomes. Nanoscale 9, 10662-10673 (2017).

25. Dou, Z. et al. $\mathrm{CO}_{2}$ fixation kinetics of Halothiobacillus neapolitanus mutant carboxysomes lacking carbonic anhydrase suggest the shell acts as a diffusional barrier for $\mathrm{CO}_{2}$. J. Biol. Chem. 283, 10377-10384 (2008).

26. Frank, S., Lawrence, A. D., Prentice, M. B. \& Warren, M. J. Bacterial microcompartments moving into a synthetic biological world. J. Biotechnol. 163, 273-279 (2013)

27. McDonald, T. M. et al. Cooperative insertion of $\mathrm{CO} 2$ in diamine-appended metal-organic frameworks. Nature 519, 303-308 (2015).

28. Bonacci, W. et al. Modularity of a carbon-fixing protein organelle. Proc. Natl Acad. Sci. USA 109, 478-483 (2012).

29. Fang, Y. et al. Engineering and modulating functional cyanobacterial $\mathrm{CO}_{2}$ fixing organelles. Front Plant Sci. 9, 739 (2018).

30. Kerfeld, C. A. \& Melnicki, M. R. Assembly, function and evolution of cyanobacterial carboxysomes. Curr. Opin. Plant Biol. 31, 66-75 (2016).

31. Wang, $\mathrm{H}$. et al. Rubisco condensate formation by CcmM in beta-carboxysome biogenesis. Nature 566, 131-135 (2019).

32. Cameron, J. C., Wilson, S. C., Bernstein, S. L. \& Kerfeld, C. A. Biogenesis of a bacterial organelle: the carboxysome assembly pathway. Cell 155, 1131-1140 (2013).

33. Chen, A. H., Robinson-Mosher, A., Savage, D. F., Silver, P. A. \& Polka, J. K. The bacterial carbon-fixing organelle is formed by shell envelopment of preassembled cargo. PLoS ONE 8, e76127 (2013).
34. Menon, B. B., Dou, Z., Heinhorst, S., Shively, J. M. \& Cannon, G. C. Halothiobacillus neapolitanus carboxysomes sequester heterologous and chimeric RubisCO species. PLoS ONE 3, e3570 (2008)

35. Iancu, C. V. et al. Organization, structure, and assembly of alphacarboxysomes determined by electron cryotomography of intact cells. J. Mol. Biol. 396, 105-117 (2010).

36. Roberts, E. W., Cai, F., Kerfeld, C. A., Cannon, G. C. \& Heinhorst, S. Isolation and characterization of the Prochlorococcus carboxysome reveal the presence of the novel shell protein CsoS1D. J. Bacteriol. 194, 787 (2012).

37. Klein, M. G. et al. Identification and structural analysis of a novel carboxysome shell protein with implications for metabolite transport. J. Mol. Biol. 392, 319-333 (2009).

38. Sawaya, M. R. et al. The structure of beta-carbonic anhydrase from the carboxysomal shell reveals a distinct subclass with one active site for the price of two. J. Biol. Chem. 281, 7546-7555 (2006).

39. Schmid, M. F. et al. Structure of Halothiobacillus neapolitanus carboxysomes by cryo-electron tomography. J. Mol. Biol. 364, 526-535 (2006).

40. Baker, S. H., Williams, D. S., Aldrich, H. C., Gambrell, A. C. \& Shively, J. M Identification and localization of the carboxysome peptide Csos3 and its corresponding gene in Thiobacillus neapolitanus. Arch. Microbiol. 173, 278-283 (2000).

41. So, A. K. et al. A novel evolutionary lineage of carbonic anhydrase ( $\varepsilon$ class) is a component of the carboxysome shell. J. Bacteriol. 186, 623-630 (2004).

42. Baker, S. H. et al. The correlation of the gene csoS2 of the carboxysome operon with two polypeptides of the carboxysome in thiobacillus neapolitanus. Arch. Microbiol. 172, 233-239 (1999).

43. Cai, F. et al. Advances in understanding carboxysome assembly in Prochlorococcus and Synechococcus implicate CsoS2 as a critical component. Life 5, 1141-1171 (2015).

44. Oltrogge, L. M. et al. Multivalent interactions between CsoS2 and Rubisco mediate alpha-carboxysome formation. Nat. Struct. Mol. Biol. 27, 281-287 (2020).

45. Chaijarasphong, T. et al. Programmed ribosomal frameshifting mediates expression of the alpha-carboxysome. J. Mol. Biol. 428, 153-164 (2016).

46. Long, B. M., Badger, M. R., Whitney, S. M. \& Price, G. D. Analysis of carboxysomes from Synechococcus PCC7942 reveals multiple Rubisco complexes with carboxysomal proteins CcmM and CcaA. J. Biol. Chem. 282, 29323-29335 (2007)

47. Stripp, S. T. \& Happe, T. How algae produce hydrogen-news from the photosynthetic hydrogenase. Dalton Trans. 38, 9960-9969 (2009).

48. Winkler, M., Esselborn, J. \& Happe, T. Molecular basis of [FeFe]-hydrogenase function: an insight into the complex interplay between protein and catalytic cofactor. Biochim. Biophys. Acta 1827, 974-985 (2013).

49. Yacoby, I. et al. Photosynthetic electron partitioning between [FeFe]hydrogenase and ferredoxin: NADP+-oxidoreductase (FNR) enzymes in vitro. Proc. Natl Acad. Sci. USA 108, 9396-9401 (2011).

50. Smith, P. R., Bingham, A. S. \& Swartz, J. R. Generation of hydrogen from $\mathrm{NADPH}$ using an [FeFe] hydrogenase. Int. J. Hydrog. Energy 37, 2977-2983 (2012).

51. King, P. W., Posewitz, M. C., Ghirardi, M. L. \& Seibert, M. Functional studies of [FeFe] hydrogenase maturation in an Escherichia coli biosynthetic system. J. Bacteriol. 188, 2163-2172 (2006).

52. Kuchenreuther, J. M., Britt, R. D. \& Swartz, J. R. New insights into [FeFe] hydrogenase activation and maturase function. PloS ONE 7, e45850 (2012).

53. Mahinthichaichan, P., Morris, D. M., Wang, Y., Jensen, G. J. \& Tajkhorshid, E. Selective permeability of carboxysome shell pores to anionic molecules. J. Phys. Chem. B 122, 9110-9118 (2018).

54. Lee, M. J., Brown, I. R., Juodeikis, R., Frank, S. \& Warren, M. J. Employing bacterial microcompartment technology to engineer a shell-free enzymeaggregate for enhanced 1, 2-propanediol production in Escherichia coli. Metab. Eng. 36, 48-56 (2016).

55. Lee, M. J. et al. Engineered synthetic scaffolds for organizing proteins within the bacterial cytoplasm. Nat. Chem. Biol. 14, 142-147 (2018).

56. Tsai, Y. et al. Structural analysis of CsoS1A and the protein shell of the Halothiobacillus neapolitanus carboxysome. PLoS Biol. 5, e144 (2007).

57. Faulkner M. et al. Molecular simulations unravel the molecular principles that mediate selective permeability of carboxysome shell protein. Sci. Rep. 10, 17501 (2020).

58. Menon, B. B., Heinhorst, S., Shively, J. M. \& Cannon, G. C. The carboxysome shell is permeable to protons. J. Bacteriol. 192, 5881-5886 (2010).

59. Sun, Y. et al. Light modulates the biosynthesis and organization of cyanobacterial carbon fixation machinery through photosynthetic electron flow. Plant Physiol. 171, 530 (2016).

60. Huang, F. et al. Roles of RbcX in carboxysome biosynthesis in the cyanobacterium Synechococcus elongatus PCC7942. Plant Physiol. 179, 184-194 (2019). 


\section{Acknowledgements}

This work was supported by the Royal Society University Research Fellowship (UF120411 and URF\R\180030, L-N.L.), the Royal Society Fellow Enhancemen Awards (RGF\EA $\backslash 181061$ and RGF\EA \180233, L-N.L.), Biotechnology and Biological Sciences Research Council Grant (BB/M024202/1 and BB/R003890/1, L.-N.L.), the British Council Newton Fund Grant Agreement PhD Placements (201703780114 to T.L., 201806370307 to J.H.), the Leverhulme Trust Early Career Fellowship ECF-2016778 (F.H.), the National Natural Science Foundation of China (91851103 and 31770128, Q.W.), the 111 Project (\#D16014, Q.W.) and the UK Engineering and Physical Sciences Research Council (EPSRC) (grant EP/N004884/1, C.M.A., R.S.S., A.I.C.). The authors would like to thank Mrs Alison Beckett for technical support of electron microscopy, the Liverpool Centre for Cell Imaging (CCI) for provision of imaging equipment and technical assistance, the Liverpool Centre for Proteome Research (CPR) for mass spectrometry and data analysis, and the Materials Innovation Factory (MIF) for provision of analytical equipment.

\section{Author contributions}

T.L., A.I.C. and L-N.L. conceived the concept. T.L., Q.J., J.H. Q.W. and H-L.H. designed and carried out the experiments. C.M.A. and R.S.S. assisted with catalyst performance evaluation and data analysis. F.H. and M.Y. assisted with confocal microscopy. G.F.D. assisted with electron microscopy. L-N.L., Q.W. and A.I.C. directed the research. T.L. and L-N.L. wrote the paper, with the contributions from all authors. All authors discussed the results.

\section{Competing interests}

T.L., Q.J., F.H. and L-N.L. are named as inventors on a patent application related to the construction and encapsulation of the carboxysome shell to improve enzyme activity. The patent application number is GB 2015810.1. The authors declare no competing interests.

\section{Additional information}

Supplementary information is available for this paper at https://doi.org/10.1038/s41467020-19280-0.

Correspondence and requests for materials should be addressed to Q.W. or L.-N.L.

Peer review information Nature Communications thanks Pamela Silver, and the other, anonymous, reviewer(s) for their contribution to the peer review of this work.

Reprints and permission information is available at http://www.nature.com/reprints

Publisher's note Springer Nature remains neutral with regard to jurisdictional claims in published maps and institutional affiliations.

(c) (i) Open Access This article is licensed under a Creative Commons Attribution 4.0 International License, which permits use, sharing, adaptation, distribution and reproduction in any medium or format, as long as you give appropriate credit to the original author(s) and the source, provide a link to the Creative Commons license, and indicate if changes were made. The images or other third party material in this article are included in the article's Creative Commons license, unless indicated otherwise in a credit line to the material. If material is not included in the article's Creative Commons license and your intended use is not permitted by statutory regulation or exceeds the permitted use, you will need to obtain permission directly from the copyright holder. To view a copy of this license, visit http://creativecommons.org/ licenses/by/4.0/.

(C) The Author(s) 2020 